\title{
Implementing mHealth-Enabled Integrated Care for Complex Chronic Patients With Osteoarthritis Undergoing Primary Hip or Knee Arthroplasty: Prospective, Two-Arm, Parallel Trial
}

Jordi Colomina ${ }^{1}$, MD; Reis Drudis ${ }^{2}, \mathrm{MD}$; Montserrat Torra ${ }^{2}, \mathrm{MD}$; Francesc Pallisó ${ }^{1}, \mathrm{PhD} ;$ Mireia Massip $^{3}, \mathrm{BScN}$; Eloisa Vargiu ${ }^{4}, \mathrm{PhD}$; Nuria Nadal ${ }^{5}, \mathrm{MD}$; Araceli Fuentes ${ }^{6}, \mathrm{MD}$; Marta Ortega Bravo ${ }^{7,8,9}$, MD; Felip Miralles ${ }^{4}$, PhD; Ferran Barbé e $^{3,10}$, MD; Gerard Torres ${ }^{3,10}$, MD; Jordi de Batlle ${ }^{3,10}$, PhD; The CONNECARE-Lleida Group ${ }^{11}$

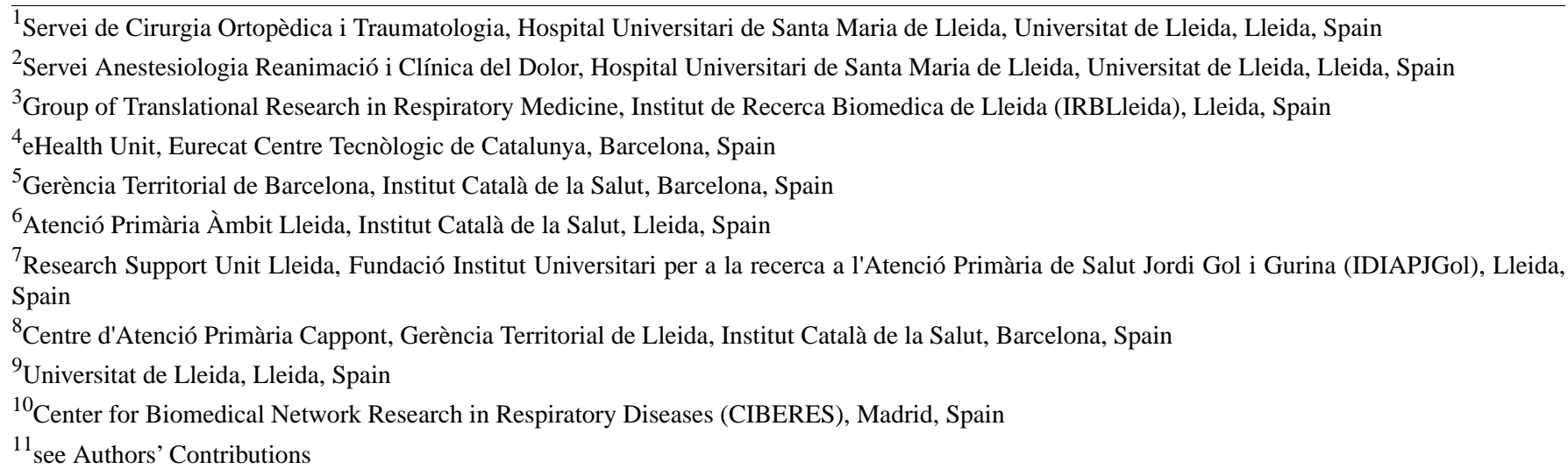

\section{Corresponding Author:}

Jordi de Batle, PhD

Group of Translational Research in Respiratory Medicine

Institut de Recerca Biomedica de Lleida (IRBLleida)

Rovira Roure 80

Lleida, 25198

Spain

Phone: 34645624734

Email: jordidebatlle@gmail.com

\section{Abstract}

Background: Osteoarthritis is a disabling condition that is often associated with other comorbidities. Total hip or knee arthroplasty is an effective surgical treatment for osteoarthritis when indicated, but comorbidities can impair their results by increasing complications and social and economic costs. Integrated care (IC) models supported by eHealth can increase efficiency through defragmentation of care and promote patient-centeredness.

Objective: This study aims to assess the effectiveness and cost-effectiveness of implementing a mobile health (mHealth)-enabled IC model for complex chronic patients undergoing primary total hip or knee arthroplasty.

Methods: As part of the Horizon 2020 Personalized Connected Care for Complex Chronic Patients (CONNECARE) project, a prospective, pragmatic, two-arm, parallel implementation trial was conducted in the rural region of Lleida, Catalonia, Spain. For 3 months, complex chronic patients undergoing total hip or knee arthroplasty and their caregivers received the combined benefits of the CONNECARE organizational IC model and the eHealth platform supporting it, consisting of a patient self-management app, a set of integrated sensors, and a web-based platform connecting professionals from different settings, or usual care (UC). We assessed changes in health status (12-item short-form survey [SF-12]), unplanned visits and admissions during a 6-month follow-up, and the incremental cost-effectiveness ratio.

Results: A total of 29 patients were recruited for the mHealth-enabled IC arm, and 30 patients were recruited for the UC arm. Both groups were statistically comparable for baseline characteristics, such as age; sex; type of arthroplasty; and Charlson index, American Society of Anesthesiologists classification, Barthel index, Hospital Anxiety and Depression scale, Western Ontario and McMaster Universities Osteoarthritis Index, and Pfeiffer mental status questionnaire scores. Patients in both groups had 
significant increases in the SF-12 physical domain and total SF-12 score, but differences in differences between the groups were not statistically significant. IC patients had $50 \%$ fewer unplanned visits $(P=.006)$. Only 1 hospital admission was recorded during the follow-up (UC arm). The IC program generated savings in different cost scenarios, and the incremental cost-effectiveness ratio demonstrated cost-effectiveness.

Conclusions: Chronic patients undergoing hip or knee arthroplasty can benefit from the implementation of patient-centered mHealth-enabled IC models aimed at empowering patients and facilitating transitions from specialized hospital care to primary care. Such models can reduce unplanned contacts with the health system and reduce overall health costs, proving to be cost-effective. Overall, our findings support the notion of system-wide cross-organizational care pathways supported by mHealth as a successful way to implement IC for patients undergoing elective surgery.

(J Med Internet Res 2021;23(9):e28320) doi: $10.2196 / 28320$

\section{KEYWORDS}

mHealth; osteoarthritis; arthroplasty; health plan implementation; chronic disease; mobile phone

\section{Introduction}

The progressive aging of populations has led to an increased burden of chronic diseases [1]. Osteoarthritis (OA) is one of the most disabling chronic diseases in developed countries. Worldwide estimates show that $10 \%$ of men and $18 \%$ of women aged >60 years have symptomatic OA [2]. Knees and hip are the most affected locations. Among the people with OA, $80 \%$ have movement limitations and $25 \%$ are disabled to perform major activities of daily living [2]. OA is associated with increased comorbidities and mortality. More than half of people with OA have another chronic medical condition, and approximately one-third of people have five or more chronic conditions [3].

Total hip arthroplasty (THA) and total knee arthroplasty (TKA) are effective surgical treatments for end-stage OA, improving joint function and health-related quality of life (QoL) [4]. Since 2000, the number of hip and knee replacements has increased rapidly in most countries in the Organization for Economic Co-operation and Development [5]. On average, hip replacement rates increased by 30\% between 2007 and 2017, and knee replacement rates increased by $40 \%$ [5]. Comorbidities are independent predictors of increased postoperative complications and nonhomebound discharge in patients undergoing shoulder, hip, or knee arthroplasty [6] and are associated with physical function and pain after primary TKA [6].

So far, the strategies aiming to improve the outcomes of elective surgeries have mainly focused on enhanced recovery protocols, prehabilitation, and postoperative rehabilitation protocols, which have been proven effective for lower limb arthroplasty [7,8]. Nevertheless, there are aspects of the entire care process that can still be improved. Traditional care models suffer from care fragmentation, with the different care levels failing to communicate effectively. After transitioning from hospital care to primary care, patients can have changes in prescribed drugs or repetition of tests because of a lack of communication across health settings [9]. Moreover, traditional models still focus on diseases rather than patients, which makes patients and their caregivers passive actors in the process [9]. Therefore, there is a need for a profound redesign of how care is provided to chronic older patients to ensure quality and sustainability [10]. Integrated care (IC) models aim to increase health efficiency through defragmentation of care, including the promotion of collaboration across settings, promotion of patient-centeredness, and prioritization of preventive strategies [11]. Interestingly, the use of eHealth tools could be the key to enabling such models [12], as demonstrated by projects such as Personalized Connected Care for Complex Chronic Patients (CONNECARE) [13] or BeyondSilos [14], which have proven the feasibility of eHealth-enabled IC in the Catalan setting. The adoption of efficient IC models can result in better outcomes for THA and TKA. In this regard, a 2017 systematic review on the benefits of telerehabilitation after orthopedic surgery showed promising results for patients undergoing THA or TKA [15]. More recently, Jonker et al [16] confirmed the feasibility of perioperative eHealth interventions for the management of older surgical patients. However, there are very few initiatives that fully embrace the use of eHealth-enabled IC models in older patients undergoing THA or TKA.

The CONNECARE project is a European Union Horizon 2020 Research and Innovation project aiming to co-design, develop, deploy, and evaluate a novel smart and adaptive organizational IC model for complex chronic patients (CCPs) [17]. From 2016 to 2019 , the project co-designed an organizational model for IC and an eHealth platform supporting it by means of an iterative patient-centered process involving patients and stakeholders across different health settings. The resulting IC model promoted collaboration among professionals in different care settings (hospital specialists, family physicians, and social workers), prioritizing home-based prevention strategies over institutional reactive care and fostering patient empowerment. A Smart Adaptive Case Management (SACM) system is offered as a web-based platform to professionals from different settings, and a patient-empowering mobile health (mHealth) self-management system allowing for three-level monitoring features and advanced communication is offered to patients.

As part of the CONNECARE project, a novel mHealth-enabled IC model was implemented in Lleida, Spain, targeting older CCPs undergoing elective THA or TKA. The existing care model for THA and TKA in Lleida is an enhanced recovery after surgery (ERAS) pathway based on different interventions to reduce perioperative stress; maintain and support homeostasis and physiological function; and accelerate the achievement of discharge criteria, including minimizing complications and readmission $[18,19]$. Although it produces good results with low transfusion, low complications, and decreased length of 
stay, it is limited by the scarce communication between professionals in different care settings, mostly hospital and primary care professionals, with different electronic medical records (EMRs) systems in hospitals and primary care centers (Argos SAP and ECAP [20], respectively). Therefore, we hypothesized that the new mHealth-enabled IC model could result in better outcomes for our patients.

This paper describes the results in terms of effectiveness and cost-effectiveness of the implementation of an mHealth-enabled IC model for the prevention of hospital readmissions in CCPs undergoing THA and TKA.

\section{Methods}

\section{Study Design}

This was a prospective, pragmatic, two-arm, parallel implementation trial comparing usual care (UC) with a 3-month mHealth-enabled IC intervention. The study was conducted from July 2018 to August 2019 at the University Hospital of Santa Maria (Lleida, Spain) and its network of primary care centers. This corresponds to a large rural area accounting for more than 236,000 citizens with a life expectancy of 80 and 86 years for men and women, respectively [21].

\section{Target Population}

The eligibility criteria were home-dwelling patients elected for primary THA or TKA at the University Hospital of Santa Maria; aged >65 years; being defined as CCP (Charlson index score $\geq 3$, taking four or more pills per day, and having had contact with the health system at least two times in the last 12 months); being classified according to the American Society of Anesthesiologists (ASA) classification as ASA II (mild systemic disease) or ASA III (severe systemic disease); and successfully passing a basic technological test, aimed to ensure the availability of internet connection at home as well as patients' or caregivers' competence with the use of a smartphone, tablet, or computer. The basic technological test can be found in Multimedia Appendix 1 [20,22].

\section{Recruitment}

The recruitment was done in several waves to match the pace of the CONNECARE project technological developments. In each wave, consecutive potential participants were contacted by a case manager during preoperative assessment at the anesthesiology outpatient clinic. The case manager explained the study protocol and obtained informed consent. These patients formed the intervention arm. After the recruitment of each patient included in the intervention arm, an active search for a control with similar characteristics from the surgery waitlist of the Orthopedics Department of University Hospital of Santa Maria began. This enhanced the similarity of patients in the intervention and control arms, although it implied a certain lag in the recruitment of controls (from some days to few weeks). All patients and their caregivers, regardless of study arm, received a face-to-face explanation about the study and provided informed consent.

\section{Intervention}

Patients in the intervention arm were attended using an IC model, including (1) preliminary assessment of the patient's health status using several questionnaires, tests, and indices specific to their main chronic diseases and social needs; (2) a self-management app, with status and performance reports, a virtual coach with customizable automated feedback, and full communication with the care team; (3) a Fitbit Flex 2 digital activity tracker [23], fully integrated into the self-management app; (4) a patient's profile in the SACM web-based platform, accessible to all members of the care team (family physicians, hospital surgical team, and social workers) and used to coordinate professionals in the different settings and enable a communication channel among them and with the patient, when needed; and (5) assignment of a case manager in charge of supervising the whole process and being the main patient contact point. Additional details on the CONNECARE IC model and the supporting eHealth platform can be found in Multimedia Appendix 1. Patients in the control arm were attended using care as usual, being managed from primary care after hospital discharge. After discharge from the initial 90 days of UC or IC management, all patients had 3 months of additional passive follow-up.

\section{Data Collection}

Variables characterizing the patients were collected at recruitment using the SACM on tablet and/or desktop computers. Collected variables included main baseline characteristics, such as age, sex, main chronic diseases, Charlson index of comorbidities [24], ASA scores [25], Barthel index for activities of daily living [26], Hospital Anxiety and Depression scale [27], assessment of the dwelling characteristics, main medications, Pfeiffer mental status questionnaire [28], and tobacco and alcohol consumption.

The cost estimation of the IC program and used health care resources is described in Multimedia Appendix 1. Briefly, IC costs included the costs of newly required medical personnel (hospital-based nurse case managers) and the costs of licensing and running the IC platform. In contrast, the one-off costs associated with the development of the IC model and supporting technology or the tuning of the system were not considered. Similarly, the required restructuring of health care professionals' time to include new tasks related to the IC model was assumed to be covered by the health system at no additional cost, as we assumed that such restructuring would not imply any additional time. Costs associated with the use of health care resources, such as medical visits and hospital admissions, were based on official data from the Catalan Health Department [22]. All costs were originally in Euros $(€)$ and were converted into US \$ (conversion used: $€ 1=U S \$ 1.21$ ).

The main outcomes were (1) intervention effectiveness, as measured by the changes in the 12-item short-form survey (SF-12) health questionnaire's physical and mental domains (baseline vs discharge) [29]; (2) use of health care resources after 6 months; and (3) cost-effectiveness, based on the improvement in QoL relative to costs, assessed by means of the incremental cost-effectiveness ratio (ICER). The source of health information was the EMR, and the collected information 
included hospital admissions, emergency room visits, visits to primary care, and visits to hospital specialists.

\section{Statistical Analyses}

Participants' baseline characteristics were described by n (\%), mean (SD), or median (P25-P75), as appropriate. Comparisons between IC and control patients' baseline characteristics were performed using the chi-square test, $t$ test, or Kruskal-Wallis test, as appropriate. A paired $t$ test comparing baseline with discharge was used to assess changes in the SF-12 domains. Linear regression models were used to assess the differences in the changes experienced by IC and control patients. Negative binomial regression models were used to assess the differences in the number of visits and admissions. Models were adjusted for age, sex, and Charlson score. ICER was calculated in relation to the SF-12 total score. Sensitivity analyses assuming two different scenarios, $150 \%$ and $200 \%$ estimated cost of the IC program, were performed to explore the cost-effectiveness performance of the IC model under unplanned increases in the implementation costs. Data analyses were conducted using Stata version 12.1 (StataCorp). The threshold for significance was set at a $P$ value of .05. All $t$ tests were two-tailed.

\section{Ethical Considerations}

This study was approved by the Ethics Committee of Hospital Arnau de Vilanova (CEIC-1685), and all patients provided written informed consent. All collected data were handled and stored in accordance with the current national and international legislation.

\section{Results}

Up to 82 patients were screened for eligibility. Of them, $49 \%$ (40/82) failed the technological test because they did not have an internet connection and 4\% (3/82) refused to participate. Therefore, 39 patients were recruited for the mHealth-enabled IC arm and 30 for the UC arm. Final analyses were based on 29 IC and 30 control patients who completed the follow-up (Figure 1).

Figure 1. Study flowchart. EMR: electronic medical record.

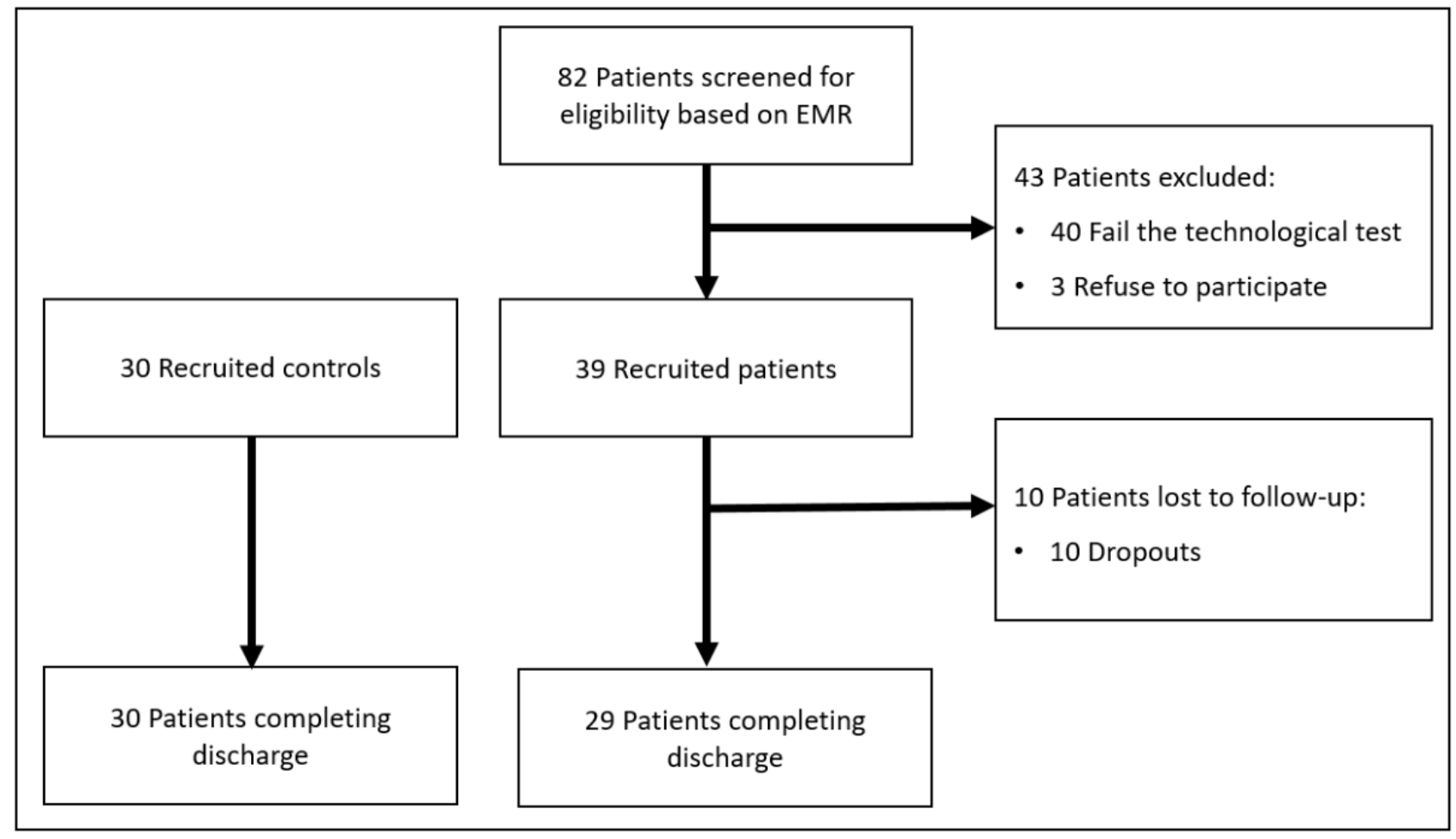

The baseline characteristics of the patients are presented in Table 1. Although the proportion of knee surgeries was slightly higher in the IC arm, no significant differences were found in the baseline characteristics of the patients in the two arms. 
Table 1. Baseline characteristics of the patients in the usual care and integrated care arms $(\mathrm{N}=59)$.

\begin{tabular}{|c|c|c|c|}
\hline Characteristic & Usual care $(\mathrm{n}=30)$ & Integrated care $(\mathrm{n}=29)$ & $P$ value $^{\mathrm{a}}$ \\
\hline Sex (male), n (\%) & $8(27)$ & $12(41)$ & .23 \\
\hline Age (years), mean (SD) & $74(8)$ & $72(9)$ & .46 \\
\hline Charlson score, mean (SD) & $4.3(1.7)$ & $4.2(1.5)$ & .81 \\
\hline \multicolumn{3}{|c|}{ American Society of Anesthesiologists class, $n(\%)$} & .52 \\
\hline II & $10(33)$ & $12(41)$ & \\
\hline III & $20(67)$ & $17(59)$ & \\
\hline \multicolumn{4}{|c|}{ Western Ontario and McMaster Universities Osteoarthritis Index score, mean (SD) } \\
\hline Pain & $9.5(3.4)$ & $9.6(3.8)$ & .93 \\
\hline Function & $38.7(12.8)$ & $39.8(14.2)$ & .77 \\
\hline Stiffness & $2.3(2.1)$ & $3.0(2.8)$ & .29 \\
\hline Barthel score, median (IQR) & $95(90-100)$ & $100(95-100)$ & .16 \\
\hline $\mathrm{HAD}^{\mathrm{b}}$ scale anxiety score, mean (SD) & $6.2(4.9)$ & $5.0(3.9)$ & .34 \\
\hline HAD scale depression score, mean (SD) & $5.0(2.3)$ & $5.1(2.9)$ & .88 \\
\hline Pfeiffer intact intellectual functioning, $\mathrm{n}(\%)$ & $30(100)$ & $27(93)$ & .14 \\
\hline Surgery location: knee, $\mathrm{n}(\%)$ & $22(73)$ & $26(90)$ & .11 \\
\hline
\end{tabular}

${ }^{\mathrm{a}}$ Chi-square test, $t$ test, or Kruskal-Wallis equality-of-populations rank test, as appropriate.

${ }^{b}$ HAD: Hospital Anxiety and Depression.

Table 2 shows the changes in QoL (SF-12 domains) from IC and control patients showed no differences. Stratification baseline to discharge. Regardless of the study arm, all patients according to the surgical location (hip or knee) reported similar showed a substantial increase in the SF-12 physical domain. findings.

The analysis of the differences in differences in QoL between

Table 2. Changes in health status in the usual care and integrated care arms.

\begin{tabular}{|c|c|c|c|c|}
\hline 12-item short-form survey score & Baseline, mean (SD) & Discharge, mean (SD) & Change, mean (SD) & $P$ value \\
\hline \multicolumn{5}{|l|}{ Physical } \\
\hline $\mathrm{UC}^{\mathrm{a}}$ & $27.9(6.4)$ & $42.0(7.7)$ & $14.1(9.0)$ & $<.001^{\mathrm{b}}$ \\
\hline $\mathrm{IC}^{\mathrm{c}}$ & $29.9(10.0)$ & $45.3(9.8)$ & $15.4(11.7)$ & $<.001^{\mathrm{b}}$ \\
\hline Difference & $2.0(2.2)$ & $3.3(2.2)$ & $1.4(3.2)$ & $.79^{\mathrm{d}}$ \\
\hline \multicolumn{5}{|l|}{ Mental } \\
\hline $\mathrm{UC}$ & $48.1(14.2)$ & $50.2(13.5)$ & $2.0(11.9)$ & $.35^{\mathrm{b}}$ \\
\hline IC & $52.1(14.2)$ & $52.8(12.9)$ & $0.8(15.2)$ & $.79^{\mathrm{b}}$ \\
\hline Difference & $3.9(3.6)$ & $2.6(3.6)$ & $-1.3(5.1)$ & $.94^{\mathrm{d}}$ \\
\hline \multicolumn{5}{|l|}{ Total } \\
\hline $\mathrm{UC}$ & 76.1 (15.6) & $92.2(18.1)$ & $16.1(14.8)$ & $<.001^{\mathrm{b}}$ \\
\hline IC & $81.9(18.8)$ & $98.1(15.6)$ & $16.2(14.3)$ & $<.001^{\mathrm{b}}$ \\
\hline Difference & $5.9(4.5)$ & $6.0(4.5)$ & $0.1(6.3)$ & $.94^{\mathrm{d}}$ \\
\hline
\end{tabular}

${ }^{\mathrm{a}} \mathrm{UC}$ : usual care.

${ }^{\mathrm{b}}$ Paired $t$ test comparing baseline and discharge measures.

${ }^{\mathrm{c}} \mathrm{IC}$ : integrated care.

${ }^{\mathrm{d}}$ Linear regression predicting the difference in the changes experienced by each arm, adjusted by age, sex, and Charlson index. 
Table 3 shows that IC patients had 50\% fewer unplanned visits than patients in the UC arm, either when considering visits directly related to the surgical procedure or any visits. One patient in the UC arm required hospital admission, whereas no admissions were recorded among IC arm patients.

Table 3. Total use of health services during the follow-up period ( $\mathrm{N}=59)$.

\begin{tabular}{|c|c|c|c|c|}
\hline Health service & $\begin{array}{l}\text { Usual care }(n=30) \text {, mean } \\
\text { (SD) }\end{array}$ & $\begin{array}{l}\text { Integrated care }(\mathrm{n}=29) \text {, mean } \\
(\mathrm{SD})\end{array}$ & $P$ value $^{\mathrm{a}}$ & Adjusted $P$ value ${ }^{\mathrm{b}}$ \\
\hline All unplanned visits & $1.4(1.5)$ & $0.7(1.0)$ & .03 & .006 \\
\hline Unplanned visits directly related to the surgery procedure & $0.8(1.2)$ & $0.4(0.7)$ & .10 & .02 \\
\hline All hospital admissions & $0.03(0.2)$ & $0(0)$ & $\mathrm{N} / \mathrm{A}^{\mathrm{c}}$ & N/A \\
\hline $\begin{array}{l}\text { Hospital admissions directly related to the surgery proce- } \\
\text { dure }\end{array}$ & $0.03(0.2)$ & $0(0)$ & N/A & N/A \\
\hline
\end{tabular}

${ }^{\mathrm{a}}$ Negative binomial regression model.

${ }^{\mathrm{b}}$ Negative binomial regression model adjusted for age, sex, and Charlson comorbidity index.

${ }^{\mathrm{c}} \mathrm{N} / \mathrm{A}$ : not applicable.

The analyses of within-trial costs and cost-effectiveness for all unplanned visits and hospital admissions are summarized in Table 4 and those for unplanned visits and hospital admissions related to the arthroplasty procedure are summarized in Table 5. The IC program generated savings from $€ 109.88$ (US $\$ 132.96$ ) to $€ 126.99$ (US $\$ 153.66$ ) per patient, depending on the nature of unplanned visits and hospitalizations. The IC program was cost-effective according to ICER, performing similar in terms of QoL gain while reducing overall expenses because of the reduction of unplanned visits and hospital admissions.

Table 4. Changes in health-related quality of life, within-trial costs (average cost per patient), and cost-effectiveness considering all unplanned visits and hospital admissions.

\begin{tabular}{|c|c|c|c|c|}
\hline Variables & Usual care $(n=30)$ & Integrated care $(n=29)$ & Difference & $\mathrm{ICER}^{\mathrm{a}}$ \\
\hline Changes in the 12-item short-form survey score, mean (SD) & $16.1(14.8)$ & $16.2(14.3)$ & $0.1(6.3)$ & $\mathrm{N} / \mathrm{A}^{\mathrm{b}}$ \\
\hline Unplanned visits $\operatorname{costs}^{\mathrm{c}}$ (US \$) & 106.07 & 51.74 & -54.33 & N/A \\
\hline Hospital admissions $\operatorname{costs}^{\mathrm{c}}$ (US \$) & 185.25 & 0 & -185.25 & N/A \\
\hline Total medical costs per patient (US \$) & 291.32 & 51.74 & -239.58 & N/A \\
\hline $\begin{array}{l}\text { Personalized Connected Care for Complex Chronic Patients program cost } \\
\text { (US \$) }\end{array}$ & 0 & 85.92 & 85.92 & N/A \\
\hline Total costs per patient (US \$) & 291.32 & 137.66 & -153.66 & -1920.73 \\
\hline
\end{tabular}

${ }^{\text {a} I C E R: ~ i n c r e m e n t a l ~ c o s t-e f f e c t i v e n e s s ~ r a t i o ; ~ i n c r e m e n t a l ~ c o s t ~ a s s o c i a t e d ~ w i t h ~ o n e ~ a d d i t i o n a l ~ p o i n t ~ g a i n ~ i n ~ t h e ~ 12-i t e m ~ s h o r t-f o r m ~ s u r v e y . ~}$

${ }^{\mathrm{b}} \mathrm{N} / \mathrm{A}$ : not applicable.

${ }^{\mathrm{c}}$ Costs based on the Catalan Institute of Health official pricing. 
Table 5. Changes in health-related quality of life, within-trial costs (average cost per patient), and cost-effectiveness considering unplanned visits and hospital admissions related to the surgical intervention.

\begin{tabular}{|c|c|c|c|c|}
\hline Variables & Usual care $(n=30)$ & Integrated care $(n=29)$ & Difference & ICER $^{\mathrm{a}}$ \\
\hline Changes in the 12-item short-form survey score, mean (SD) & $16.1(14.8)$ & $16.2(14.3)$ & $0.1(6.3)$ & $\mathrm{N} / \mathrm{A}^{\mathrm{b}}$ \\
\hline Unplanned visits costs ${ }^{\mathrm{c}}$ (US \$) & 64.68 & 31.05 & -33.63 & N/A \\
\hline Hospital admissions $\operatorname{costs}^{\mathrm{c}}$ (US \$) & 185.25 & 0 & -185.25 & N/A \\
\hline Total medical costs per patient (US \$) & 249.93 & 31.05 & -218.88 & N/A \\
\hline $\begin{array}{l}\text { Personalized Connected Care for Complex Chronic Patients program cost } \\
\text { (US \$) }\end{array}$ & 0 & 85.92 & 85.92 & N/A \\
\hline Total costs per patient (US \$) & 249.93 & 116.97 & -132.96 & -1661.94 \\
\hline
\end{tabular}

${ }^{\text {a}}$ ICER: incremental cost-effectiveness ratio; incremental cost associated with one additional point gain in the 12-item short-form survey.

${ }^{\mathrm{b}} \mathrm{N} / \mathrm{A}$ : not applicable.

${ }^{\mathrm{c}}$ Costs based on the Catalan Institute of Health official pricing.

Sensitivity analyses assuming two different cost scenarios, $150 \%$ and $200 \%$ estimated cost of the IC program, thus exploring cost-effectiveness under unplanned increases in the implementation costs, showed savings and cost-effectiveness, as shown in Tables S1 and S2 in Multimedia Appendix 1.

\section{Discussion}

\section{Principal Findings}

The prospective assessment of the implementation of an mHealth-enabled IC program for TKA and THA management showed a reduction in the number of unplanned contacts with the health system after the surgery; generated substantial savings for the health system, while not having any negative impact on QoL or clinical outcomes; and demonstrated cost-effectiveness.

\section{Strengths and Limitations}

The implemented IC model had several strengths that must be highlighted. First, there was an effort to involve all the stakeholders from different organizations that would be actors in a large-scale deployment of the mHealth-enabled IC program since the very early stages. This is key, as the lack of cooperation among professionals, teams, and organizations is a recurrent barrier for effective IC implementation [11]. Second, informal caregivers played a role in the IC process, as close relatives of the patients can be their link with the health system. Moreover, informal caregivers can play a key role in facilitating the use of self-management apps in older patients. Third, the self-management app for patients (allowing active monitoring, communication with the care team, and a virtual coach with customizable automated feedback) enhanced doctor-patient relationships [30] and facilitated early detection of any problem in the surgical recovery process [31]. Fourth, the assessment and promotion of patients' physical activity is an effective way of preventing any mobility impairment, which is found in one-third of people aged $>65$ years [32]. Finally, the region selected for the deployment of the IC program, a large rural area, was especially appropriate as their citizens can benefit the most from community-based IC initiatives that can avoid unnecessary travel to the hospital.
Regarding this study, several strengths and limitations should be noted. Among the strengths, we note the use of a prospective study design with a comparator arm; the use of objectively measured endpoints, such as visits and admissions, in contrast to patient-reported outcomes; and cost and cost-effectiveness assessments. Concerning the limitations, the technological platform supporting the implemented IC model showed substantial improvements throughout the implementation period. This implied that patients recruited near the end of the implementation study had a richer IC experience than those recruited at the very beginning. Similarly, this had an impact on health care professionals, who had to cope with a platform under development and not fully integrated with existing EMRs. Nevertheless, participating patients and professionals showed great acceptability of the IC model and setting [33]. Moreover, directly participating in a dynamic development and implementation process fostered professionals' engagement and allowed them to propose changes and new features to be developed, which resulted in not a single professional dropping out of the study. Next, the limited number of patients involved in the study had an impact on statistical power. Nevertheless, the current number of participants sufficed to show a significant reduction in robust endpoints, such as unplanned visits and hospital admissions, and showed the cost-effectiveness of the IC program. Finally, only direct costs were considered, although the inclusion of indirect or societal costs would most likely favor the cost-effectiveness of the IC model.

\section{Comparison With Existing Literature}

This study aimed to assess the impact of the implementation of an IC model in three domains: (1) patients' QoL, (2) patients' use of health services, and (3) health economics. Regarding the QoL domain, the IC model performed as good as the UC arm in the differences-in-differences analysis. This result is in line with the mixed results found in a 2017 review on the impact of IC interventions on QoL [34]. However, it is worth noting that the great increase in QoL obtained after a successful THA or TKA is likely to mask any minor increase in QoL caused by being managed in an IC model. Next, regarding the use of health services, patients in the IC model required 50\% fewer unplanned visits after the surgery. This corresponds to the upper margin of benefits reported in reviews about IC interventions between 
2000 and 2015, which reported significant reductions in hospital activity ranging from $15 \%$ to $50 \%$ [35]. These excellent results could be in line with the notion of system-wide cross-organizational care pathways as a successful way to implement IC, in contrast to smaller and narrow interventions [9]. Finally, regarding health economics, IC generated savings from $€ 109.88$ (US \$132.96) to $€ 126.99$ (US \$153.66) per patient and was deemed cost-effective. This is in line with reviews stating the potential cost-effectiveness of IC in the management of chronic diseases [36]. However, these savings are lower than the range of US \$584-\$1434 obtained when applying the same IC model to CCPs with chronic obstructive pulmonary disease or heart failure [13], who are more prone to experiencing unplanned visits and hospitalizations.

When specifically focusing on THA and TKA, previous studies have suggested the usefulness of the different potential components of an eHealth IC model, including telerehabilitation [15], care pathways [18,37], education [38], patient-centeredness [39], mHealth continuous monitoring [40,41], and cross-setting integration [42]. However, to our knowledge, this study is the first to include all these components in a single mHealth-enabled IC model. Overall, our results are similar to or better than those reported in these previous studies, suggesting a moderate additive effect of combining the reported interventions into a single mHealth-enabled IC model.

\section{Implications for Research and/or Practice}

The World Health Organization has already stated the need for patient-centered IC models to satisfy the health needs of older populations with chronic diseases while keeping costs sustainable [29,43]. With this premise in mind, the CONNECARE project tackled the task of iteratively co-designing a mHealth-enabled IC model with the participation of all key stakeholders: patients; hospital-based surgeons, anesthesiologists, nurses, physiotherapists, and case managers; primary care physicians and nurses; social caregivers; and managers, technical staff, developers, and researchers. This multidisciplinary team envisioned a system-wide cross-organizational patient-centered care pathway, in line with the principles of the 2015 World Report on Ageing and Health [1]. The experience acquired during the co-design and testing process allows us to highlight some key features that future IC models for the management of older citizens undergoing THA or TKA should consider (1) a common cross-setting web-based platform is key for a successful coordination of care across settings and patient monitoring; (2) the habilitation of functional communication channels for the patients can be a key source of savings, as most savings are achieved through the avoidance of unplanned visits; and (3) involving informal caregivers, such as younger family members, can facilitate the adoption of mHealth tools, such as sensors and self-management apps, and make the overall user experience very satisfactory [44]. Moreover, it is key to ensure access to an internet connection at home, as this was the main criterion halting the participation of potential users.

\section{Conclusions}

The implementation of a patient-centered mHealth-enabled IC model for the management of patients undergoing THA or TKA successfully empowered patients, effectively connected the different care settings involved, reduced unplanned contacts with the health system, reduced health costs, and was cost-effective. This supports the use of mHealth tools for the implementation of system-wide cross-organizational IC models.

\section{Acknowledgments}

This work was supported by the European Union's Horizon 2020 Research and Innovation Program (under grant agreement GA-689802). JDB acknowledges receiving financial support from the Catalan Health Department (Pla Estratègic de Recerca $i$ Innovació en Salut 2016: SLT002/16/00364) and Instituto de Salud Carlos III (Miguel Servet 2019: CP19/00108), which is cofunded by the European Social Fund, Investing in your future. Funding organizations had no role in the design and conduct of the study; collection, management, analysis, and interpretation of the data; preparation, review, or approval of the manuscript; and decision to submit the manuscript for publication. The authors would like to express their sincere gratitude to all the participating patients and their caregivers as well as to all the involved health and social care professionals. All of them were key in the co-design of the integrated care model and its implementation in the region of Lleida.

\section{Authors' Contributions}

JDB, EV, NN, FP, FM, FB, and GT participated in the conceptualization of the project. JC, RD, MT, MM, EV, AF, MOB, and GT conducted the data collection. MM, EV, and JDB participated in the data curation. JDB conducted all statistical analyses. JC and JDB wrote the original draft of the manuscript. All authors reviewed the final manuscript. JDB, EV, FM, FB, and GT secured funding for the project. The CONNECARE-Lleida Group consists of: Maria Aguilà Balastegui, Sandra Alexandre Loxano, Laila Al-Jouja Llorente, Tomás Alonso Sancho, Enrique Aparicio Bañeres, Ana Arce Vila, Jose Maria Baron Burriel, Ramon Bascompte Claret, Albert Bigorda Sague, Emilia Blanco Ponce, Maria Boldú Franque, Àngels Bosch Roig, Carmen Bravo Santiago, Alba Capdevila Sarramona, Aida Castelló Corretge, Montse Coma Gassó, Fina Cregenzan Ortiz, Dolors Del Pozo Garcia, Mireia Falguera Vilamajó, Pere Farre Pagés, Yolanda Fauria Garcia, Anabel Fusalba Canales, Jara Gayan Ordas, Sergi Godia Lopez, Irene Gomez Companys, Jessica Gonzàlez Gutierrez, Anna Gort Oromí, Carme Jorge Tufet, Mercé Lavega Llorens, Laia Llort Samsó, Maria Rosa Lopez Cervelló, Belen Malla Clua, Josep Maria Marsol Mas, Teresita Martí Ribes, Diana Martin Capella, José Maria Martínez Barriuso, Esther Mateus Solé, Ramon Mazana Novellon, Petra Merino De los Santos, Miquel Mesas Julio, Sonia Minguet Vidal, Nuria Moles Porta, Luis Miguel Montaña Esteban, Dolors Morera Roset, Meritxell Moyà Oro, Irene Muñoz Del Campo, Francisco Nicolás Sánchez, Inés Ortiz Catalán, Mireia Ortiz Valls, Sonia Ortiz Congost, Jose Maria Palacin Peruga, 
Eugeni Paredes Costa, Pablo Pastor Pueyo, Ana Pérez Sainz, Antonio Plana Blanco, Anna Planas Hiraldo, Pepita Pont Aldoma, Marife Quelle Alonso, Rebeca Ramirez Molinero, Maria Àngels Revés Juanbaro, Anna Ribé Miró, Eva Ribó Caubet, Rebeca Rodriguez Corbaton, Marina Rué Florensa, Oscar Sacristán García, Irene Sanmartí Forns, Maria Cruz Sanz Martinez, Neus Sendra Bordes, Maria Cruz Urgelés Castillón, Laia Utrillo Montagut, and Montse Vidal Ballesté.

\section{Conflicts of Interest}

None declared.

\section{Multimedia Appendix 1}

Supplementary information. [DOCX File, 1565 KB-Multimedia Appendix 1]

\section{References}

1. World Health Organization. World Report on Ageing and Health. Geneva: World Health Organization; 2015:1-246.

2. Safiri S, Kolahi AA, Smith E, Hill C, Bettampadi D, Mansournia MA, et al. Global, regional and national burden of osteoarthritis 1990-2017: a systematic analysis of the Global Burden of Disease Study 2017. Ann Rheum Dis 2020 Jun;79(6):819-828. [doi: 10.1136/annrheumdis-2019-216515] [Medline: 32398285]

3. Osteoarthritis: a serious disease, submitted to the U.S. Food and Drug Administration. Osteoarthritis Research Society International. 2016. URL: https://oarsi.org/sites/default/files/library/2018/pdf/oarsi white paper oa serious disease121416 1. pdf [accessed 2021-02-13]

4. Ethgen O, Bruyère O, Richy F, Dardennes C, Reginster J. Health-related quality of life in total hip and total knee arthroplasty. A qualitative and systematic review of the literature. J Bone Joint Surg Am 2004 May;86(5):963-974. [doi: 10.2106/00004623-200405000-00012] [Medline: 15118039]

5. Organisation for Economic Cooperation and Development (OECD). Health at a Glance 2019: OECD Indicators. Paris: OECD Publishing; 2019:1-243.

6. Hilton ME, Gioe T, Noorbaloochi S, Singh JA. Increasing comorbidity is associated with worsening physical function and pain after primary total knee arthroplasty. BMC Musculoskelet Disord 2016 Oct 07;17(1):421 [FREE Full text] [doi: 10.1186/s12891-016-1261-y] [Medline: 27717340]

7. Saunders R, Seaman K, Ashford C, Sullivan T, McDowall J, Whitehead L, et al. An ehealth program for patients undergoing a total hip arthroplasty: protocol for a randomized controlled trial. JMIR Res Protoc 2018 Jun 12;7(6):e137 [FREE Full text] [doi: 10.2196/resprot.9654] [Medline: 29895515]

8. Nunns M, Shaw L, Briscoe S, Thompson CJ, Hemsley A, McGrath JS, et al. Multicomponent hospital-led interventions to reduce hospital stay for older adults following elective surgery: a systematic review. Health Serv Deli Res 2019;7(40):07400. [doi: 10.3310/hsdr07400] [Medline: 31869021]

9. Harvey G, Dollard J, Marshall A, Mittinty MM. Achieving integrated care for older people: shuffling the deckchairs or making the system watertight for the future? Int J Health Policy Manag 2018 Apr 01;7(4):290-293 [FREE Full text] [doi: 10.15171/ijhpm.2017.144] [Medline: 29626395]

10. World Health Organization. Integrated Care for Older People: Guidelines on Community-Level Interventions to Manage Declines in Intrinsic Capacity. Geneva: World Health Organization; 2017:1-46.

11. Dates M, Lennox-chhugani N, Sant H, Pereira A, Tedeschi M. Health System Performance Assessment - Integrated Care Assessment. Brussels, Belgium: European Commission; 2018:1-108.

12. Chouvarda IG, Goulis DG, Lambrinoudaki I, Maglaveras N. Connected health and integrated care: toward new models for chronic disease management. Maturitas 2015 Sep;82(1):22-27. [doi: 10.1016/j.maturitas.2015.03.015] [Medline: 25891502]

13. de Batlle J, Massip M, Vargiu E, Nadal N, Fuentes A, Bravo MO, CONNECARE-Lleida Group. Implementing mobile health-enabled integrated care for complex chronic patients: intervention effectiveness and cost-effectiveness study. JMIR Mhealth Uhealth 2021 Jan 14;9(1):e22135 [FREE Full text] [doi: 10.2196/22135] [Medline: 33443486]

14. Piera-Jiménez J, Daugbjerg S, Stafylas P, Meyer I, Müller S, Lewis L, et al. BeyondSilos, a telehealth-enhanced integrated care model in the domiciliary setting for older patients: observational prospective cohort study for effectiveness and cost-effectiveness assessments. JMIR Med Inform 2020 Oct 06;8(10):e20938 [FREE Full text] [doi: 10.2196/20938] [Medline: $\underline{\text { 33021490] }}$

15. Pastora-Bernal JM, Martín-Valero R, Barón-López FJ, Estebanez-Pérez MJ. Evidence of benefit of telerehabitation after orthopedic surgery: a systematic review. J Med Internet Res 2017 Dec 28;19(4):e142 [FREE Full text] [doi: 10.2196/jmir.6836] [Medline: 28455277]

16. Jonker LT, Haveman ME, de Bock GH, van Leeuwen BL, Lahr MM. Feasibility of perioperative eHealth interventions for older surgical patients: a systematic review. J Am Med Dir Assoc 2020 Dec;21(12):1844-1851 [FREE Full text] [doi: 10.1016/j.jamda.2020.05.035] [Medline: $\underline{\text { 32694000] }}$

17. The CONNECARE project. URL: http://www.connecare.eu [accessed 2021-02-13] 
18. Wainwright TW. Consensus statement for perioperative care in total hip replacement and total knee replacement surgery: Enhanced Recovery After Surgery (ERAS®) Society recommendations. Acta Orthop 2020 Jun 14;91(3):363 [FREE Full text] [doi: $\underline{10.1080 / 17453674.2020 .1724674}$ ] [Medline: $\underline{32056486}$ ]

19. Jones EL, Wainwright TW, Foster JD, Smith JR, Middleton RG, Francis NK. A systematic review of patient reported outcomes and patient experience in enhanced recovery after orthopaedic surgery. Ann R Coll Surg Engl 2014 Mar;96(2):89-94 [FREE Full text] [doi: 10.1308/003588414X13824511649571] [Medline: 24780662]

20. eCAP. Department of Health. URL: https://salutweb.gencat.cat/ca/ambits_actuacio/linies_dactuacio/ tecnologies informacio i comunicacio/ecap/ [accessed 2021-02-13]

21. CatSalut Memòria 2018 Regió Sanitària Lleida. Generalitat de Catalunya. URL: https://catsalut.gencat.cat/ca/coneix-catsalut/ catsalut-territori/lleida/publicacions/\#bloc1 [accessed 2021-02-13]

22. Resolution SLT/353/2013, of February 13, on the review of public prices for the services provided by the Catalan Health Institute. CVE-DOGC-A-13051031-2013. URL: https://portaldogc.gencat.cat/utilsEADOP/PDF/6326/1287494.pdf [accessed 2021-02-13]

23. Fitbit. URL: https://www.fitbit.com/pr/flex2 [accessed 2021-02-13]

24. Charlson ME, Pompei P, Ales KL, MacKenzie CR. A new method of classifying prognostic comorbidity in longitudinal studies: development and validation. J Chronic Dis 1987;40(5):373-383. [doi: 10.1016/0021-9681(87)90171-8] [Medline: $\underline{3558716}]$

25. Mayhew D, Mendonca V, Murthy BV. A review of ASA physical status - historical perspectives and modern developments. Anaesthesia 2019 Mar;74(3):373-379 [FREE Full text] [doi: 10.1111/anae.14569] [Medline: 30648259]

26. Collin C, Wade DT, Davies S, Horne V. The Barthel ADL Index: a reliability study. Int Disabil Stud 1988;10(2):61-63. [doi: 10.3109/09638288809164103] [Medline: $\underline{\text { 3403500] }}$

27. Zigmond AS, Snaith RP. The hospital anxiety and depression scale. Acta Psychiatr Scand 1983 Jun;67(6):361-370. [doi: 10.1111/j.1600-0447.1983.tb09716.x] [Medline: $\underline{\text { 6880820] }}$

28. Pfeiffer E. A short portable mental status questionnaire for the assessment of organic brain deficit in elderly patients. $\mathrm{J}$ Am Geriatr Soc 1975 Oct;23(10):433-441. [doi: 10.1111/j.1532-5415.1975.tb00927.x] [Medline: 1159263]

29. Ware J, Kosinski M, Keller SD. A 12-item short-form health survey: construction of scales and preliminary tests of reliability and validity. Med Care 1996 Mar;34(3):220-233. [doi: 10.1097/00005650-199603000-00003] [Medline: $\underline{\text { 8628042] }}$

30. Józsa T. The relevance of mobile applications helping in doctor-patient relationships. Br J Gen Pract 2020 Mar;70(692):109 [FREE Full text] [doi: 10.3399/bjgp20X708377] [Medline: $\underline{\text { 32107220] }}$

31. Aznar-Tortonda V, Palazón-Bru A, la Rosa DM, Espínola-Morel V, Pérez-Pérez BF, León-Ruiz AB, et al. Detection of frailty in older patients using a mobile app: cross-sectional observational study in primary care. Br J Gen Pract 2020 Jan;70(690):29-35 [FREE Full text] [doi: 10.3399/bjgp19X706577] [Medline: 31685541]

32. Mitra S, Sambamoorthi U. Disability prevalence among adults: estimates for 54 countries and progress toward a global estimate. Disabil Rehabil 2014;36(11):940-947. [doi: 10.3109/09638288.2013.825333] [Medline: 23962193]

33. de Batlle J, Massip M, Vargiu E, Nadal N, Fuentes A, Bravo MO, CONNECARE-Lleida Group. Implementing mobile health-enabled integrated care for complex chronic patients: patients and professionals' acceptability study. JMIR Mhealth Uhealth 2020 Nov 20;8(11):e22136 [FREE Full text] [doi: 10.2196/22136] [Medline: 33216004]

34. Flanagan S, Damery S, Combes G. The effectiveness of integrated care interventions in improving patient quality of life (QoL) for patients with chronic conditions. An overview of the systematic review evidence. Health Qual Life Outcomes 2017 Sep 29;15(1):188 [FREE Full text] [doi: 10.1186/s12955-017-0765-y] [Medline: 28962570]

35. Damery S, Flanagan S, Combes G. Does integrated care reduce hospital activity for patients with chronic diseases? An umbrella review of systematic reviews. BMJ Open 2016 Dec 21;6(11):e011952 [FREE Full text] [doi: 10.1136/bmjopen-2016-011952] [Medline: 27872113]

36. Cronin J, Murphy A, Savage E. Can chronic disease be managed through integrated care cost-effectively? Evidence from a systematic review. Ir J Med Sci 2017 Nov;186(4):827-834. [doi: 10.1007/s11845-017-1600-5] [Medline: 28477328]

37. Mertes SC, Raut S, Khanduja V. Integrated care pathways in lower-limb arthroplasty: are they effective in reducing length of hospital stay? Int Orthop 2013 Jun;37(6):1157-1163 [FREE Full text] [doi: 10.1007/s00264-013-1829-1] [Medline: 23456085]

38. McDonald S, Page MJ, Beringer K, Wasiak J, Sprowson A. Preoperative education for hip or knee replacement. Cochrane Database Syst Rev 2014 May 13(5):CD003526. [doi: 10.1002/14651858.CD003526.pub3] [Medline: 24820247]

39. Olsson L, Karlsson J, Berg U, Kärrholm J, Hansson E. Person-centred care compared with standardized care for patients undergoing total hip arthroplasty - a quasi-experimental study. J Orthop Surg Res 2014 Oct 09;9:95 [FREE Full text] [doi: 10.1186/s13018-014-0095-2] [Medline: 25359278]

40. Ramkumar PN, Muschler GF, Spindler KP, Harris JD, McCulloch PC, Mont MA. Open mHealth architecture: a primer for tomorrow's orthopedic surgeon and introduction to its use in lower extremity arthroplasty. J Arthroplasty 2017 Apr;32(4):1058-1062. [doi: 10.1016/j.arth.2016.11.019] [Medline: 27956125]

41. Ramkumar PN, Haeberle HS, Ramanathan D, Cantrell WA, Navarro SM, Mont MA, et al. Remote patient monitoring using mobile health for total knee arthroplasty: validation of a wearable and machine learning-based surveillance platform. $\mathrm{J}$ Arthroplasty 2019 Oct;34(10):2253-2259. [doi: 10.1016/j.arth.2019.05.021] [Medline: $\underline{31128890]}$ 
42. Bethge M, Bartel S, Streibelt M, Lassahn C, Thren K. [Improved outcome quality following total knee and hip arthroplasty in an integrated care setting: results of a controlled study]. Rehabilitation (Stuttg) 2011 Apr;50(2):86-93. [doi: 10.1055/s-0030-1265144] [Medline: 21503861]

43. Desmedt M, Vertriest S, Hellings J, Bergs J, Dessers E, Vankrunkelsven P, et al. Economic impact of integrated care models for patients with chronic diseases: a systematic review. Value Health 2016;19(6):892-902 [FREE Full text] [doi: 10.1016/j.jval.2016.05.001] [Medline: 27712719]

44. Vargiu E, Dorado A, Massip M, Gómez-Martínez M, Torres G, de Batlle J, et al. User experience in CONNECARE: results and lessons learnt in Lleida. XPA \& Health Communication. 2019. URL: http://www.xpabcn.com/revista/index.php/XPAHC/ article/view/22 [accessed 2021-02-13]

\author{
Abbreviations \\ ASA: American Society of Anesthesiologists \\ CCP: complex chronic patient \\ CONNECARE: Personalized Connected Care for Complex Chronic Patients \\ EMR: electronic medical record \\ ERAS: enhanced recovery after surgery \\ IC: integrated care \\ ICER: incremental cost-effectiveness ratio \\ mHealth: mobile health \\ OA: osteoarthritis \\ QoL: quality of life \\ SACM: smart adaptive case management \\ SF-12: 12 -item short-form survey \\ THA: total hip arthroplasty \\ TKA: total knee arthroplasty \\ UC: usual care
}

Edited by R Kukafka; submitted 01.03.21; peer-reviewed by G Carot-Sans, J Roca; comments to author 27.03.21; revised version
received 17.05.21; accepted 14.06.21; published 02.09.21
Please cite as:
Colomina J, Drudis R, Torra M, Pallisó F, Massip M, Vargiu E, Nadal N, Fuentes A, Ortega Bravo M, Miralles F, Barbé F, Torres
G, de Batlle J, The CONNECARE-Lleida Group
Implementing mHealth-Enabled Integrated Care for Complex Chronic Patients With Osteoarthritis Undergoing Primary Hip or Knee
Arthroplasty: Prospective, Two-Arm, Parallel Trial
J Med Internet Res 2021;23(9):e28320
URL: $\underline{\text { https://www.jmir.org/2021/9/e28320 }}$
doi: $10.2196 / 28320$
PMID: $\underline{34473068}$

CJordi Colomina, Reis Drudis, Montserrat Torra, Francesc Pallisó, Mireia Massip, Eloisa Vargiu, Nuria Nadal, Araceli Fuentes, Marta Ortega Bravo, Felip Miralles, Ferran Barbé, Gerard Torres, Jordi de Batlle, The CONNECARE-Lleida Group. Originally published in the Journal of Medical Internet Research (https://www.jmir.org), 02.09.2021. This is an open-access article distributed under the terms of the Creative Commons Attribution License (https://creativecommons.org/licenses/by/4.0/), which permits unrestricted use, distribution, and reproduction in any medium, provided the original work, first published in the Journal of Medical Internet Research, is properly cited. The complete bibliographic information, a link to the original publication on https://www.jmir.org/, as well as this copyright and license information must be included. 